\title{
ESTIMACIÓN DE UN MODELO ECONOMÉTRICO QUE RELACIONA EL DELITO DE CONTRABANDO CON LA DEVALUACIÓN DEL PESO COLOMBIANO Y SOL PERUANO
}

\section{Estimation of an econometric model that relates the crime of contraband with the devaluation of the Colombian peso and Peruvian sol}

\author{
Mauricio Abril- Donoso*, Nancy Chariguamán - Maurisaca \\ Escuela Superior Politécnica del Chimborazo, Riobamba, Ecuador \\ *mauricio.abrildo@espoch.edu.ec
}

El objetivo del estudio es poder explicar el comportamiento del delito de contrabando, en función de las devaluaciones de las monedas de Colombia y Perú. La información se recopiló de las denuncias de contrabando de la base de delitos de la Fiscalía General del Estado de Ecuador (FGE), se recabó también las cotizaciones mensuales en promedio alcanzadas por el Peso colombiano de la Superintendencia Financiera de Colombia (www.superfinanciera.gov.co) y por el sol peruano de la página web investing.com (https://es.investing.com/currencies/usd-pen-historical-data). Los resultados muestran la correcta estimación del modelo econométrico para el contrabando, además de como las variables explicativas generan el cometimiento del delito de contrabando, esto permitirá que las autoridades gubernamentales puedan tomar medidas económicas para proteger el mercado interno y externo del País

Palabras claves: contrabando, devaluaciones, modelo econométrico.

The objective of the study is to explain the behavior of the crime of contraband, based on the devaluations of the Colombian and Peruvian currencies. The information was collected from the allegations of contraband of the base of crimes of the Fiscalía General de Estado de Ecuador (FGE), the monthly quotes were also collected on average reached by the Colombian Peso of the Financial Superintendence of Colombia (www.superfinanciera.gov.co) and the Peruvian sol of the website investing.com (https://es.investing.com/currencies/usd-pen-historical-data). The results show the correct estimation of the econometric model for the contraband, in addition to how the explanatory variables generate the commission of the crime of contraband, this will allow the governmental authorities to take economic measures to protect the internal and external market of the Country.

Key words: contraband, devaluations, econometric model.

Fecha de recepción: 13-12-2018

\section{INTRODUCCIÓN}

A partir del segundo semestre del 2015 en el Ecuador se tiene una desaceleración de la economía producto de la caída del precio del petróleo y de la apreciación del dólar (Ecuadorinmediato. [Internet], 2016), lo que genera un fuerte impacto en la economía del Ecuador, así el índice de pobreza en junio de 2014 redujo en $24 \%$, mientras que en junio de 2015 redujo el 22\% (Ecuadorinmediato. [In-
Fecha de aceptación: 11-06-2019
Este fenómeno afectó también a nuestros vecinos Colombia y Perú quienes adoptaron medidas económicas para minimizar el impacto en sus economías, la principal medida implementada fue la devaluación de sus monedas locales, que a diferencia de nuestro país no podemos 
adoptarla ya que nuestra moneda oficial es el Dólar americano.

Las acciones tomadas por nuestros vecinos tienen un impacto directo en nuestra economía, ya que un efecto directo de la devaluación es la apreciación del Dólar respecto a estas monedas, esto a su vez genera un incremento en el precio de los productos en el Ecuador respecto a Colombia y Perú, ante esto en septiembre de 2015 entró en vigencia la aplicación de salvaguardas por balanza de pagos a los bienes (Ecuadorinmediato. [Internet], 2016).

Todo esto suponemos impacta en el cometimiento del delito de Contrabando (ver figura 1), ya que es rentable la venta de productos provenientes de Perú y Colombia en nuestro País, además de que muchas familias se proveen de productos básicos en Colombia y Perú muchos cometiendo sin conocimiento el delito de contrabando.

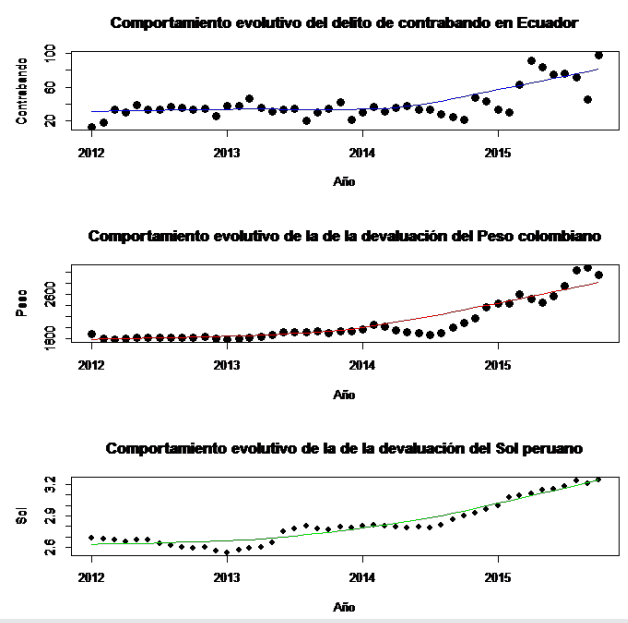

Figura 1. Evolución del Contrabando; devaluación del peso colombiano, Sol peruano respecto al Dólar.

En la figura 1 se muestra el comportamiento evolutivo de las variables que intervendrán en la estimación del modelo econométrico, como variable dependiente la evolución de las denuncias del delito de Contrabando, mientras que como variables explicativas las devaluaciones de monedas de Perú y Colombia. Con esto, el modelo pretende estimar la relación que existe entre el contrabando en función de la devaluación del peso colombiano y del sol peruano.

Para el procesamiento de la información se utilizará el paquete estadístico " $\mathrm{R}$ ", mientras que para la estimación, corrección y validación del modelo econométrico con el uso del paquete lmtest de " $\mathrm{R}$ ".

\section{MATERIALES Y MÉTODOS.}

El modelo que se quiere estimar es del siguiente tipo (Gujarati, 2010):

$$
y_{t}=\beta_{1}+\beta_{2} x_{1 t}+\beta_{3} x_{2 t}+u_{t}
$$

Que es la expresión del modelo lineal que relaciona una variable dependiente con dos variables independientes más un error, donde:

$$
\begin{gathered}
y_{t}=\text { Contrabando } \\
x_{1 t}=\text { Devaluación peso } \\
x_{2 t}=\text { Devaluaciónsol }
\end{gathered}
$$

En un inicio se estima el modelo lineal para luego hacer el diagnóstico sobre los parámetros estimados y probar la validez de este, la prueba de hipótesis sobre los coeficientes es la siguiente:

$$
\left\{H_{0}: \beta=0\right.
$$

\begin{tabular}{|lllll|}
\hline Coeficientes & Estimación & Error estándar & Valor $-\mathrm{t}$ & $\operatorname{Pr}(>|\mathrm{t}|)$ \\
\hline Intercepción & -44.57476 & 51.77805 & -0.861 & 0.3941 \\
\hline Peso Colombiano & 0.03542 & 0.01626 & 2.179 & 0.0349 \\
\hline Sol Peruano & 4.07405 & 29.22545 & 0.139 & 0.8898 \\
\hline Error estándar residual: 13.09 & con 43 grados de libertad & \\
\hline R-cuadrado: & 0.5351, & R-cuadrado ajustado: & 0.5135 \\
\hline Estadístico-F: 24.75 con 2 y 43 GL. & p-valor: & 1.233e-09 \\
\hline
\end{tabular}

Tabla 1. Parámetros del modelo lineal con variable dependiente Contrabando

La tabla 1 muestra las estimaciones por medio de mínimos cuadrados ordinarios (Wooldridge, 2010) de los parámetros del modelo planteado, con sus estadísticos para probar la hipótesis nula de que todos los parámetros son cero. Se muestra también el coeficiente de correlación del modelo el cual es de 0,5135 un coeficiente algo aceptable.

\begin{tabular}{|l|l|l|l|l|l|}
\hline Estimadores & GL & $\begin{array}{l}\text { Suma de } \\
\text { Cuadrados }\end{array}$ & $\begin{array}{l}\text { Media de Suma } \\
\text { de Cuadrados }\end{array}$ & $\begin{array}{l}\text { Valor } \\
\mathrm{F}\end{array}$ & $\operatorname{Pr}(>\mathrm{F})$ \\
\hline $\begin{array}{l}\text { Peso Co- } \\
\text { lombiano }\end{array}$ & 1 & 8480.3 & 8480.3 & 49.47 & $1.2 \mathrm{e}-08$ \\
\hline Sol Peruano & 1 & 3.3 & 3.3 & 0.02 & 0.8898 \\
\hline Residuos & 43 & 7371.0 & 171.4 & & \\
\hline
\end{tabular}

Tabla 2. Análisis de Varianza y suma de cuadrados del modelo estimado 
En la tabla 2 se muestra el análisis de varianza del modelo estimado, se observa que se tiene valores altos para las sumas de cuadrados esperadas del peso colombiano y para la suma de cuadrados de los residuos, sin embargo, la prueba $\mathrm{F}$ es significativa a un nivel del $5 \%$. Este comportamiento hace suponer que se tiene que realizar pruebas para identificar problemas de heteroscedasticidad y autocorrelación (Hansen, 2016).

Esta prueba muestra que la única variable que se relaciona con el contrabando es la devaluación del peso colombiano ya que tiene un p-valor menor al 5\%, que indica que se rechaza la hipótesis nula para este parámetro, los otros dos parámetros intersección y el parámetro para la devaluación del sol peruano no rechazan la hipótesis nula ya que su p-valor es mayor a 5\%. Continuando con la validación del modelo, se presenta el comportamiento de los residuos en los siguientes gráficos.

La figura 2 muestra el comportamiento de los residuos en relación con los valores estimados de la variable dependiente y con los residuos estandarizados, además de los gráficos de normalidad y de las distancias de los residuos a los valores de la variable dependiente. Es claro que los residuos no se comportan aleatoriamente ni tampoco siguen una distribución normal.
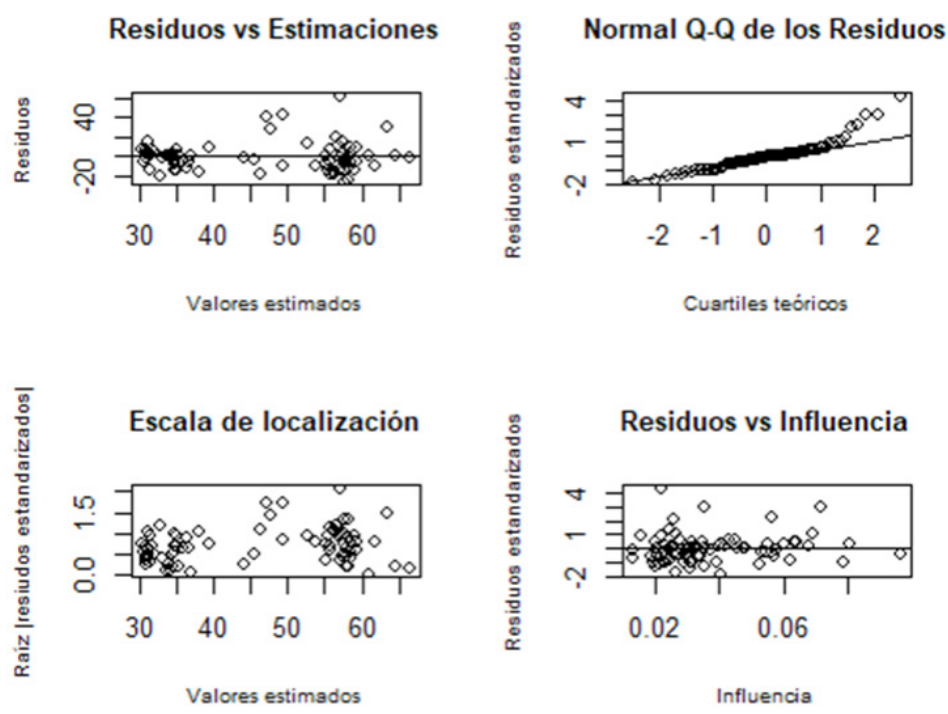

Figura 2. Comportamiento de los residuos, pruebas aleatorias

Esto indica la posible presencia de heteroscedasticidad, por lo cual se realizan algunas pruebas para probar la hipótesis de ausencia de heteroscedasticidad y poder corregirla.
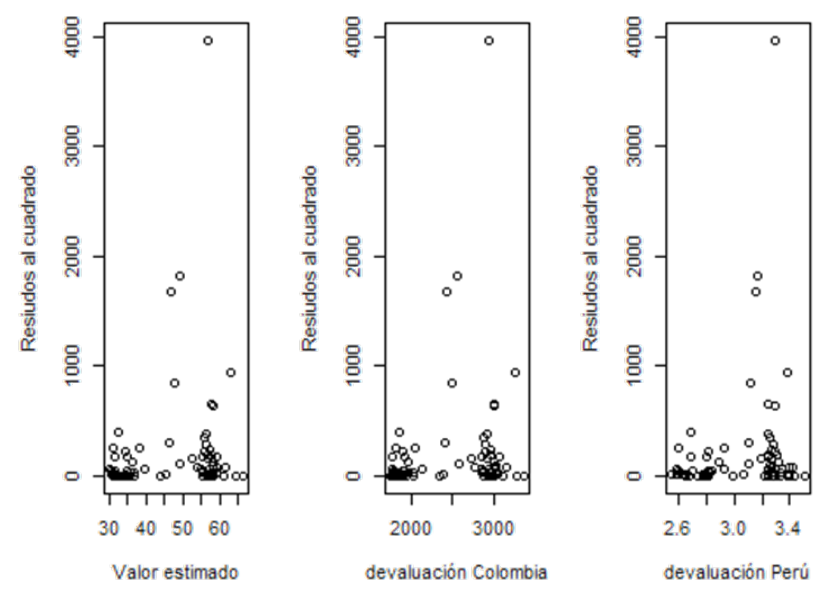

Figura 3. Análisis de los residuos según variables dependiente y explicativas.
La figura 3 muestra como los residuos al cuadrado se distribuyen, con los valores estimados del modelo, y las variables explicativas devaluación del peso colombiano y devaluación del sol peruano.

Según este análisis los residuos crecen según aumenta los valores estimados del contrabando, y las devaluaciones del peso y el sol, cabe resaltar que con el peso colombiano aumenta mucho más los residuos al cuadrado, aunque la relación con la devaluación del sol es más definida.

Se analizan dos contrastes para detectar la posible presencia de heteroscedasticidad. 


\begin{tabular}{|c|c|c|}
\hline CONTRASTE & DESCRIPCIÓN & $\begin{array}{c}\text { PRUEBA Y P-VA- } \\
\text { LOR }\end{array}$ \\
\hline $\begin{array}{l}\text { Goldfeld y } \\
\text { Quandt (1965) }\end{array}$ & $\begin{array}{l}\text { Supone que } \sigma_{t}^{2} \text { depende de } z_{t} \\
\text { El contraste consiste en: } \\
\text { a) Ordenar las observaciones de } z_{t} \text { de mayor a menor. } \\
\text { b) } \quad \text { Omitir p observaciones de la mitad de la muestra. } \\
\text { c) } \quad \text { Estimar dos veces el modelo original con las } \frac{T-p}{2} \text { variables iniciales y finales. } \\
\text { d) } \\
\text { Si el valor del estadístico excede el valor de la tabla se rechaza la hipótesis nula. }\end{array}$ & $\begin{array}{l}\mathrm{GQ}=3.3419 \\
\mathrm{Gl} 1=20 \\
\mathrm{Gl} 2=20 \\
\text { p-valor }=0.004791\end{array}$ \\
\hline $\begin{array}{l}\text { Breusch y } \\
\text { Pagan }\end{array}$ & $\begin{array}{l}\text { Suponemos que: } \sigma_{t}^{2}=h\left(z_{t}^{\prime} \alpha\right)=h\left(\alpha_{0}+\alpha_{1} z_{1 t}+\alpha_{2} z_{2 t}+\cdots+\alpha_{p} z_{p t}\right) \\
\text { Se prueba la hipótesis } H_{0}: \alpha_{1}=\alpha_{2}=\cdots \alpha_{p}=0 \\
\text { Se efectúa como sigue: } \\
\text { a) Estimar por MCO y obtener los residuos. } \\
\text { b) Se normalizan los residuos } \hat{e}_{t}^{2}=\frac{\hat{u}_{t}^{2}}{\hat{\sigma}_{u}^{2}} t=1,2, \cdots T \\
\text { c) Se estima la regresión de } \hat{e}_{t}^{2} \text { sobre una constante y } z_{1 t}, z_{2 t}, \cdots z_{p t} \text { se tiene } \\
\text { ST=SE+SR } \\
\text { d) Se estima SE/2 se distribuye con una } X_{p}^{2} \\
\text { Si el valor del estadístico t es mayor que el punto crítico, se rechaza la hipótesis nula. }\end{array}$ & $\begin{array}{l}\mathrm{BP}=16.704 \\
\mathrm{Gl}=2 \\
\mathrm{p} \text {-valor }=0.0002359\end{array}$ \\
\hline
\end{tabular}

Tabla 3. Contrastes para detectar la hetroscedasticidad con paquete lmtest de $\mathrm{R}$

Todos los contrastes que se presentan en la tabla 3 , indican presencia de heteroscedasticidad, como el de Breush y Pagan (Greene, 2012) ahora lo que sigue es estimar la forma de la heteroscedasticidad, que variables la producen para poder corregirla.

Para esto vamos a utilizar el contraste de Glesjer (Novales, 1993), el cual además de identificar la presencia de heteroscedasticidad, trata también de identificar la verdadera estructura de esta, no limitándose a estructuras lineales.

Para esto se estima la siguiente regresión de los residuos sobre una variable explicativa.

$\left|\widehat{u_{t}}\right|=\delta_{0}+\delta_{1} z_{t}^{h}+v_{t}$

Esto para distintos valores de $h$ : $h=\left\{-1,1, \frac{1}{2},-\frac{1}{2}\right\}$

Con esto se escoge el valor de $h$ que proporcione la mejor regresión.

Una vez que se han probado los modelos para todos los posibles valores de $\mathrm{h}$, tanto para la devaluación del peso colombiano como del sol peruano, se tiene que los mejores modelos se tienen para $h=-1$ para el peso colombiano y para $h=1$ para el sol peruano.

De estos el que mejor se comporta según los estimadores, es el que se estimó para el peso colombiano, con este modelo se corrige el modelo estimado inicial y se tiene los nuevos estimadores MCG del modelo inicial.

Se realizan las siguientes transformaciones sobre las variables originales, del modo siguiente:

$\mathrm{W} 1=$ contrabando/estimación (2)

$\mathrm{Z1}$ 1=1/estimación (2)

$\mathrm{Z} 2=$ devaluación peso/estimación (2) 
Z3=devaluación sol/estimación (2)

Con lo que se estima el modelo siguiente:

\begin{tabular}{|lclll|}
\hline Coeficientes & Estimación & Error estándar & Valor $-\mathrm{t}$ & $\operatorname{Pr}(>|\mathrm{t}|)$ \\
\hline Z1 & 47.45373 & 32.94542 & 1.44 & 0.157004 \\
\hline Z2 & 0.06126 & 0.01505 & 4.07 & 0.000198 \\
\hline Z3 & -47.63045 & 20.27074 & -2.35 & 0.023449 \\
\hline Error estándar residual: 1.205 con 43 grados de libertad \\
\hline R-cuadrado: & 0.9487, & R-cuadrado ajustado: 0.9451 \\
\hline Estadístico-F: 265.1 con 3 y 43 GL. & p-valor: 2.2e-16 \\
\hline
\end{tabular}

En la tabla 4 se muestra los estimadores del modelo corregido, en la misma se observa que los estimadores de la devaluación del Peso colombiano y la devaluación del Sol peruano ya son estadísticamente significativos y rechaza la hipótesis nula de que estos son cero. De igual forma el valor de R-cuadrado mejora considerablemente, por lo que se tiene un modelo aceptable.

\begin{tabular}{|l|l|l|l|l|l|}
\hline Estimadores & GL & $\begin{array}{l}\text { Suma de } \\
\text { Cuadrados }\end{array}$ & $\begin{array}{l}\text { Media de } \\
\text { Suma de } \\
\text { Cuadrados }\end{array}$ & Valor F & $\operatorname{Pr}(>\mathrm{F})$ \\
\hline Z1 & 1 & 1122.08 & 1122.08 & 772.6 & $2.24 \mathrm{e}-16$ \\
\hline Z2 & 1 & 25.04 & 25.04 & 17.2 & 0.00015 \\
\hline Z3 & 1 & 8.02 & 8.02 & 5.5 & 0.02344 \\
\hline Residuos & 43 & 62.45 & 1.45 & & \\
\hline
\end{tabular}

Tabla 5. Tabla ANOVA del modelo transformado

En la tabla 5 se presenta las sumas de cuadrados para las variables y los residuos, la cual indica que son todas estadísticamente significativas, que ratifica la valides del modelo estimado. Se puede observar que los valores de las sumas esperadas de Colombia y de los residuos disminuyen considerablemente.

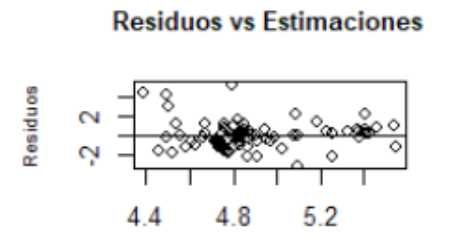

Valores estimados

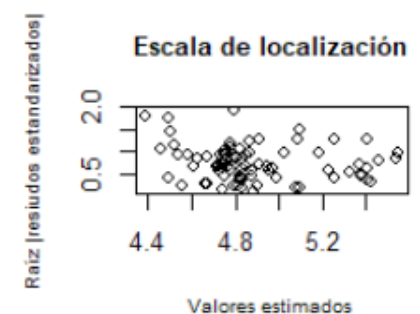

Figura 4. Residuos del modelo corregido heteroscedasticidad
El nuevo modelo estimado que se corrigió la presencia de heteroscedasticidad, según la metodología propuesta por Glesjer, aplicando las pruebas de heteroscedasticidad del paquete lmtest de $\mathrm{R}$ (Thomson, 2018), observamos que ya no se rechaza la hipótesis nula de que el modelo es homoscedastico como se muestra en la tabla6.

$\mathrm{BP}=1.7415, \mathrm{GL}=2$, Valor- $\mathrm{p}=0.4186$

Hipótesis alternativa: Heteroscedasticidad es mayor que 0

Tabla 6. Prueba de Breusch-Pagan para heteroscedasticidad

Una vez que se ha corregido la heteroscedasticidad, se investiga el nivel de autocorrelación de los errores mediante el método de Durbin-Watson (Hanke, 1996).

$d=\frac{\sum_{t=2}^{n}\left(\widehat{u_{t}}-\widehat{u_{t-1}}\right)^{2}}{\sum_{t=1}^{n} \hat{u}_{t}^{2}}$

Con esto se trata de estimar un modelo autorregresivo de primer orden de los errores correlacionados.

$u_{t}=\rho u_{t-1}+\varepsilon_{t}$

Donde:

$\hat{\rho}=\frac{\sum \hat{u}_{t} \hat{u}_{t-1}}{\sum \hat{u}_{t}^{2}}$

Expresando el estimador $d$ en términos de $\hat{\rho}$ tenemos

$d \approx 2(1-\hat{\rho})$

Pero, como $-1 \leq \rho \leq 1 \Rightarrow 0 \leq d \leq 4$

En el paquete lmtest de $\mathrm{R}$ se tiene también este contraste para identificar el nivel de autocorrelación y si este influye en la validez del modelo estimado y que esta corregido de heterocedasticidad. 
DW $=1.2667$, Valor- $\mathrm{p}=0.001857$

Hipótesis alternativa: La verdadera autocorrelación es mayor que 0

Tabla 7. Prueba de autocorrelación de los errores.

Aplicando la prueba de Durbin-Watson se observa que se rechaza la hipótesis nula de ausencia de autocorrelación, bajo estas hipótesis se tiene que corregir la autocorrelación estimando una regresión sobre los residuos y estimando el coeficiente de autocorrelación de primer orden.

\begin{tabular}{|c|c|c|c|c|}
\hline Coeficientes & Estimación & Error estándar & Valor- & $t \operatorname{Pr}(>|t|)$ \\
\hline ET1 & 0.2803 & 0.1328 & 2.11 & 0.0406 \\
\hline \multicolumn{5}{|c|}{ Error estándar residual: 1.038 con 44 grados de libertad } \\
\hline \multicolumn{5}{|c|}{ R-cuadrado: 0.09187, R-cuadrado ajustado: 0.07123} \\
\hline \multicolumn{5}{|c|}{ Estadístico-F: 4.451 con 1 y 44 GL. p-valor: 0.0406} \\
\hline
\end{tabular}

Tabla 8. Coeficiente de correlación para AR(1)

Con la estimación del coeficiente de primer orden se procede a transformar las variables originales y realizar una nueva estimación de los coeficientes, las transformaciones sugeridas son las siguientes:

$$
\begin{aligned}
& w t t=w 1_{t}-0.28 * w 1_{t-1} \\
& v 0 t t=z 1_{t}-0.28 * z 1_{t-1} \\
& v 1 t t=z 2_{t}-0.28 * z 2_{t-1} \\
& v 2 t t=z 3_{t}-0.28 * z 3_{t-1}
\end{aligned}
$$

\begin{tabular}{|c|c|c|c|c|}
\hline Coeficientes & Estimación & Error estándar & : Valor-t & $\operatorname{Pr}(>|t|)$ \\
\hline V0TT & 38.25459 & 38.52959 & 0.993 & 0.32646 \\
\hline $\mathrm{Z} 2$ & 0.05579 & 0.01711 & 3.260 & 0.00221 \\
\hline $\mathrm{Z3}$ & -40.10102 & 23.25522 & -1.724 & 0.09199 \\
\hline \multicolumn{5}{|c|}{ Error estándar residual: 1.085 con 42 grados de libertad } \\
\hline \multicolumn{5}{|c|}{ R-cuadrado: 0.9285 , R-cuadrado ajustado: 0.9234} \\
\hline
\end{tabular}

Con estas transformaciones se procede a estimar el nuevo modelo como sigue.

Tabla 9. Estimación del modelo para corregir la autocorrelación

Se tienen una nueva estimación de los coeficientes del modelo transformado.
DW $=1.9644$, Valor-p $=0.3394$

Hipótesis alternativa: La verdadera autocorrelación es mayor que 0

Tabla 10. Prueba de errores del modelo corregido

Con la prueba ya no se rechaza la hipótesis nula de ausencia de autocorrelación, ya que el valor del estadístico es próximo a 2 y con un $\mathrm{p}$-valor de 0,34 , esto indica que el modelo estimado está libre de autocorrelación.
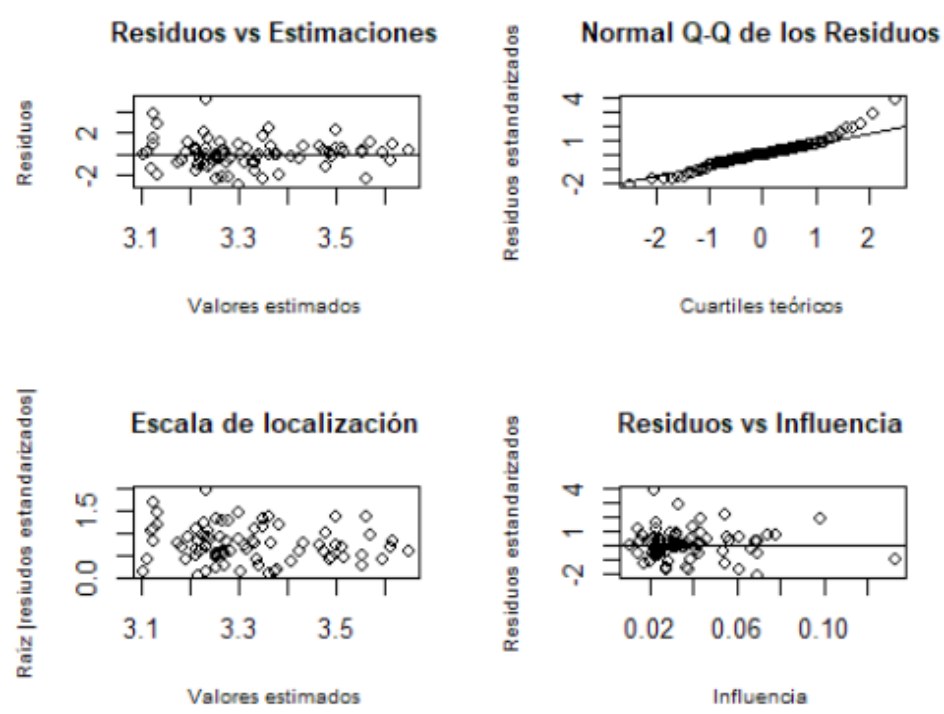

Figura 5. Residuos del modelo corregido autocorrelación

En la figura 5 se muestra cómo se distribuyen los residuos del modelo estimado corregido de autocorrelación, la misma que confirma los resultados y pruebas de los estimadores y nos permite validar el modelo estimado.

\section{RESULTADOS}

Con esto el modelo que mejor ajusta la estimación del contrabando en términos de la devaluación del peso colombiano y la devaluación del sol peruano, corregido tanto de heteroscedasticidad como de autocorrelación es el siguiente:

$\widehat{\text { contrabando }}=38,25+0,056 * \widehat{\text { colombia }}-40,1 * \widehat{\text { perú }}+u_{t}(7)$

Modelo que como se mostró en el apartado anterior supera las pruebas estadísticas y se tiene el modelo validado, con esto se puede indicar que:

- Se tiene una relación lineal del delito de contrabando con la devaluación de las monedas de Colombia y Perú, las cuales intervienen en el cometimiento de este delito. 
- Cuando Colombia devalúa su moneda en 100 pesos el contrabando se incrementa en 6 casos adicionales, mientras que, si Perú devalúa su moneda en 100 Pesos, se reduciría el contrabando en 40 puntos.

- Este fenómeno puede deberse a que si los dos estimadores son positivos el contrabando crecería infinitamente, lo cual no es verdad y claro el país que más ha devaluado la moneda ha sido Colombia.

\section{DISCUSIÓN}

El contrabando al ser un delito internacional y que obedece a generar economías ilegales (Fundación Konrad Adenauer, 2015), es claro que tiene que ser evidenciado y descubierto al igual que otros delitos como el narcotráfico, tráfico de armas, tráfico de combustibles, etc., esto es no se tiene víctimas afectadas por este delito, sino que el afectado es el Estado ecuatoriano.

De los estimadores que se obtuvo se pueden realizar las siguientes consideraciones para que el descubrimiento y judicialización tenga mejores resultados (Contrabando, 2015):

- Se puede relacionar el contrabando con otras variables que pueden también influir especialmente en frontera sur limítrofe con el Perú, donde se el estimador si este país aumenta la devaluación de su moneda local, el contrabando disminuiría, esto puede deberse, a que por reportes de fiscalía por esta frontera se tiene un mayor delito de tráfico de combustibles como gasolina, Diesel y gas doméstico. Lo que podría ser más rentable económicamente para quienes ejecutan estos delitos.

- Otro aspecto que se debe tener en cuenta es la Política Tributaria que se tiene en cada País limítrofe con el Ecuador.

- Sería importante el estudiar los productos y mercancías que más se contrabandean en las fronteras y determinar su influencia en la estimación del modelo.
- Se debe considerar es la geografía de las dos fronteras, la extensión de la frontera y las distancias a las ciudades donde se llevará la mercadería de contrabando para su comercialización.

\section{CONCLUSIONES}

- El contrabando es un delito económico, como tal obedece en gran parte al comportamiento de la economía nacional, este se incrementa con la apreciación del dólar americano, dado que los precios de artículos importados suben en nuestros países, mientras que en nuestros vecinos Colombia y Perú los mismos productos tienen menor precio, ya que al tener moneda propia pueden devaluar la misma.

- Para minimizar el cometimiento de este delito en el País, es necesario implementar salvaguardas en el mercado nacional desde el campo económico.

- Dado que se tiene un número importante de personas que adquieren productos en Colombia y Perú, y el limitado personal aduanero no abastece en el control y descubrimiento de este y otros delitos en frontera sur y norte, se tiene que implementar medidas económicas que permitan tributar sobre los artículos y productos que se ingrese a nuestro País.

- Se tiene que realizar un seguimiento sobre indicadores económicos internacionales, de forma que se pueda tener una reacción adecuada sobre el comportamiento fluctuante y dinámico del mercado internacional. 


\section{$\mathrm{R}$ eferencias}

1. Contrabando. Fiscalía General del Estado. 2015. 2015, Perfil Criminológico, pág. 16.

2. Ecuadorinmediato. 2016. Ecuador afectado por crisis económica mundial, Gobierno privilegia inversión a creación de fondos de ahorro. ECUADORINMEDIATO.com. [En línea] 01 de 01 de 2016. Disponible en: http://ecuadorinmediato.com/index.php?module=Noticias\&func=news user_view\&id=2818793964\&umt=ecuador_afectado_por_crisis_economica_mundial_gobierno_ privilegia_inversion_a_creacion_fondos_ahorro.

3. Fundación Konrad Adenauer. 2015. Red Centroamericana de Centros de Pensamiento e Incidencia - LA RED. [En línea] 01 de Febrero de 2015. www.kas.de/c/document_library/get_file?uui$\mathrm{d}=$ f5461a20-d8fc-44ce-7071-c4bba9b0274d\&groupId=252038.

4. Greene, William. 2012. Econometric Analisis . Boston : Prentice Hall, 2012.

5. Gujarati, D, Porter D. 2010. Econometría. Mexico : McGrawHill, 2010.

6. Hanke, J \& Reitsch, A. 1996. Pronosticos en los Negocios. Mexico : Pearson, 1996.

7. Hansen, Bruce. 2016. Econometrics. Wisconsin : University of Wisconsin, 2016.

8. Novales, Alfonso. 1993. Econometría. segunda. Madrid : Mc Grawm Hill, 1993.

9. Thomson, Hothorn. 2018. Testing Linear Regression Models Package 'lmtest' by R. Testing Linear Regression Models Package 'lmtest' by R. [En línea] 04 de 04 de 2018. https://cran.r-project. org/web/packages/lmtest/lmtest.pdf.

10. Wooldridge, Jeffrey. 2010. Introducción a la Econometría. Murcia : Thomson, 2010. 